\title{
Classification of Swift's gamma-ray bursts
}

\author{
I. Horváth ${ }^{1}$, L. G. Balázs ${ }^{2}$, Z. Bagoly ${ }^{3}$, and P. Veres ${ }^{1,3}$ \\ 1 Dept. of Physics, Bolyai Military University, POB 15, 1581 Budapest, Hungary \\ e-mail: horvath.istvan@zmne.hu \\ 2 Konkoly Observatory, POB 67, 1525 Budapest, Hungary \\ 3 Dept. of Physics of Complex Systems, Eötvös University, Pázmány P. s. 1/A, 1117 Budapest, Hungary \\ Received 27 May 2008 / Accepted 1 August 2008
}

\begin{abstract}
Context. Two classes of gamma-ray bursts have been identified in the BATSE catalogs characterized by durations shorter and longer than about $2 \mathrm{~s}$. There are, however, some indications for the existence of a third class. Swift satellite detectors have different spectral sensitivity than pre-Swift ones for gamma-ray bursts. Therefore we reanalyze the durations and their distribution and also the classification of GRBs.

Aims. We analyze the bursts duration distribution, published in The First BAT Catalog, whether it contains two, three or more groups. Methods. Using The First BAT Catalog the maximum likelihood estimation was used to analyze the duration distribution of GRBs. Results. The three log-normal fit is significantly (99.54\% probability) better than the two for the duration distribution. Monte-Carlo simulations also confirm this probability (99.2\%). Similarly, in previous results we found that the fourth component is not needed. The relative frequencies of the distribution of the groups are $7 \%$ short $35 \%$ intermediate and $58 \%$ long.

Conclusions. Similarly to the BATSE data, three components are needed to explain the BAT GRBs' duration distribution. Although the relative frequencies of the groups are different than in the BATSE GRB sample, the difference in the instrument spectral sensitivities can explain this bias. This means theoretical models may be needed to explain three different type of gamma-ray bursts.
\end{abstract}

Key words. gamma rays: bursts - gamma rays: theory - gamma rays: observations

\section{Introduction}

It has been a great challenge to classify gamma-ray bursts (GRBs). Mazets et al. (1981) and Norris et al. (1984) suggested there might be a separation in their duration distribution. Using The First BATSE Catalog, Kouveliotou et al. (1993) found a bimodality in the distribution of the logarithms of the durations. In that paper they used the parameter $T_{90}$ (the time in which $90 \%$ of the fluence is accumulated, Kouveliotou et al. 1993) to characterize the duration of GRBs (McBreen et al. 1994; Koshut et al. 1996; Belli 1997; Pendleton et al. 1997). Today it is widely accepted that the physics of these two groups (also called "subclasses" or simply "classes") are different, and these two kinds of GRBs are different phenomena (Norris et al. 2001; Balázs et al. 2003; Fox et al. 2005). In the Swift database the measured redshift distribution for the two groups are also different, for short bursts the median is 0.4 (O'Shaughnessy et al. 2008) and for the long ones it is 2.4 (Bagoly et al. 2006).

The bimodal distribution was further quantified in another paper (Kouveliotou et al. 1996), where a two-log-normal fit was made; the best parameters of the fit were published in McBreen et al. (1994) and Koshut et al. (1996).

In a previous paper using the Third BATSE Catalog (Meegan et al. 1996) Horváth (1998) showed that the duration $\left(T_{90}\right)$ distribution of GRBs observed by BATSE could be well fitted by a sum of three log-normal distributions. We find it statistically unlikely (with a probability $\sim 10^{-4}$ ) that there are only two groups. Simultaneously, Mukherjee et al. (1998) report the finding (in a multidimensional parameter space) of a very similar group structure of GRBs. Somewhat later, several authors (Hakkila et al. 2000; Balastegui et al. 2001;
Rajaniemi \& Mähönen 2002; Hakkila et al. 2003; Borgonovo 2004; Hakkila et al. 2004; Chattopadhyay et al. 2007) included more physical parameters in the analysis of the bursts (e.g. peak-fluxes, fluences, hardness ratios, etc.). A cluster analysis in this multidimensional parameter space suggests the existence of the third ("intermediate") group as well (Mukherjee et al. 1998; Hakkila et al. 2000; Balastegui et al. 2001; Rajaniemi \& Mähönen 2002; Chattopadhyay et al. 2007). The physical existence of the third group is, however, still not convincingly proven. However, the celestial distribution of the third group is anisotropic (Mészáros et al. 2000; Litvin et al. 2001; Magliocchetti et al. 2003). All these results mean that the existence of the third intermediate group in the BATSE sample is acceptable, but its physical meaning, importance and origin is less clear than those of the other groups. Hence, it is worth studying new samples if their size is large enough for statistical analysis. In the HETE-II database (Vanderspek et al. 2004) there are only 104 GRBs and in the Swift first BAT database (Sakamoto et al. 2008) there are 237 GRBs. Therefore, in this paper we use the Swift data because of its better statistics.

In Sect. 2 we discuss the method used in the paper. In Sect. 3 uni-, bi-, tri- and tetra-modal log-normal fits made by using the maximum likelihood method are discussed. In Sect. 4 one thousand Monte-Carlo simulations are shown investigating the significance of the fits. In Sect. 5 we discuss some further details. The conclusions are given in Sect. 6 .

\section{The method}

There are several methods to test significance. For example the $\chi^{2}$ method which we used in our first paper (Horváth 1998) to 


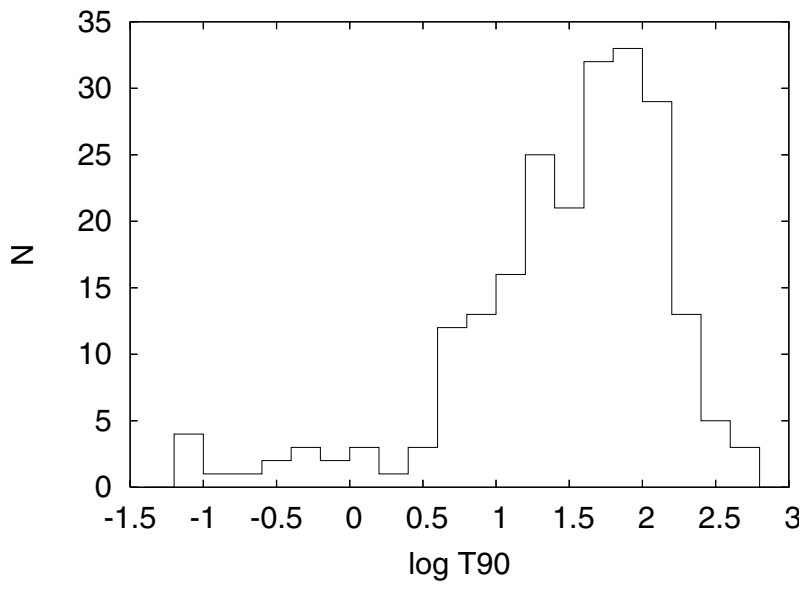

Fig. 1. Duration distribution of the observed BAT bursts.

analyze the $T_{90}$ distribution of the BATSE bursts is not useful here, because of the small population of short bursts in the Swift sample.

In the Swift BAT Catalog (Sakamoto et al. 2008) there are 237 GRBs, of which 222 have duration information. Figure 1 shows the $\log T_{90}$ distribution. To use the $\chi^{2}$ method one has to bin the data. If the number of counts within some bins is small the method is not applicable. The Maximum Likelihood (ML) method is not sensitive to this problem, therefore for the (Swift) BAT bursts the maximum likelihood method is much more appropriate.

The ML method assumes that the probability density function of an $x$ observable variable is given in the form of $g\left(x, p_{1}, \ldots, p_{k}\right)$ where $p_{1}, \ldots, p_{k}$ are parameters of unknown value. Having $N$ observations of $x$ one can define the likelihood function in the following form:

$l=\prod_{i=1}^{N} g\left(x_{i}, p_{1}, \ldots, p_{k}\right)$

or in logarithmic form (the logarithmic form is more convenient for calculations):

$L=\log l=\sum_{i=1}^{N} \log \left(g\left(x_{i}, p_{1}, \ldots, p_{k}\right)\right)$.

The ML procedure maximizes $L$ according to $p_{1}, \ldots, p_{k}$. Since the logarithmic function is monotonic the logarithm reaches the maximum where $l$ does as well. The confidence region of the estimated parameters is given by the following formula, where $L_{\max }$ is the maximum value of the likelihood function and $L_{0}$ is the likelihood function at the true value of the parameters (Kendall \& Stuart 1973):

$2\left(L_{\max }-L_{0}\right) \approx \chi_{k}^{2}$

\section{Log-normal fits of the duration distribution}

Similarly to Horváth (2002) we fit the $\log T_{90}$ distribution using ML with a superposition of $k$ log-normal components, each of them having 2 unknown parameters to be fitted with $N=222$ measured points in our case. Our goal is to find the minimum value of $k$ suitable to fit the observed distribution. Assuming a
Table 1. The best parameters for the two log-normal fit of the GRB duration distribution.

\begin{tabular}{cccc}
\hline \hline & Duration $\left(\log T_{90}\right)$ & $\sigma\left(\log T_{90}\right)$ & $w$ \\
\hline short & -0.456 & 0.501 & 16.3 \\
long & 1.606 & 0.507 & 205.7 \\
\hline
\end{tabular}

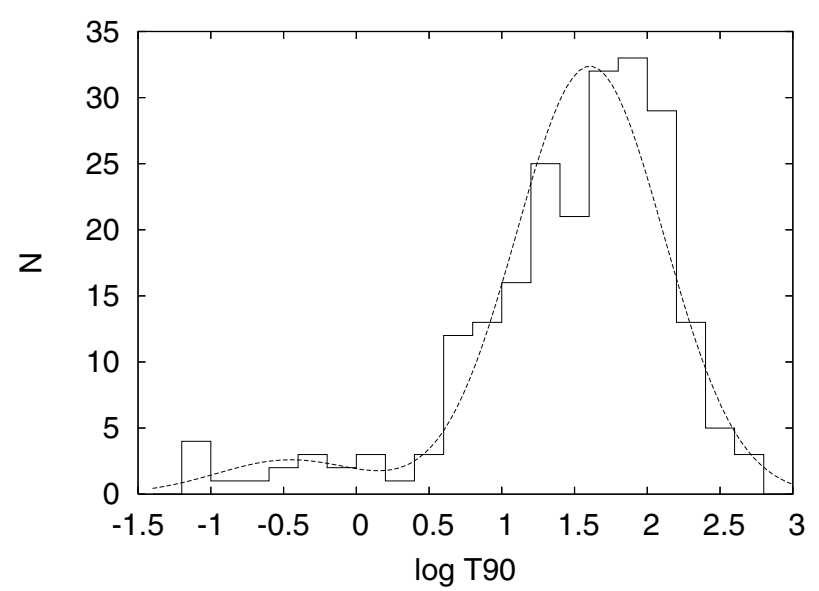

Fig. 2. Fit with two log-normal component for the duration distribution of BAT bursts.

weighted superposition of $k$ log-normal distributions one has to maximize the following likelihood function:

$L_{k}=\sum_{i=1}^{N} \log \left(\sum_{l=1}^{k} w_{l} f_{l}\left(x_{i}, \log T_{l}, \sigma_{l}\right)\right)$

where $w_{l}$ is a weight, $f_{l}$ a $\log$-normal function with $\log T_{l}$ mean and $\sigma_{l}$ standard deviation having the form of

$f_{l}=\frac{1}{\sigma_{l} \sqrt{2 \pi}} \exp \left(-\frac{\left(x-\log T_{l}\right)^{2}}{2 \sigma_{l}^{2}}\right)$

and due to a normalization condition

$\sum_{l=1}^{k} w_{l}=N$.

We used a simple $\mathrm{C}++$ code to find the maximum of $L_{k}$. Assuming only one log-normal component the fit gives $L_{1 \max }=$ 951.666 but in the case of $k=2$ one gets $L_{2 \max }=983.317$ with the parameters given in Table 1 and the solution displayed in Fig. 2.

Based on Eq. (3) we can infer whether the addition of a further log-normal component is necessary to signifincantly improve the fit. We take the null hypothesis that we have already reached the the true value of $k$. Adding a new component, i.e. moving from $k$ to $k+1$, the ML solution of $L_{k \max }$ change to $L_{(k+1) \max }$, but $L_{0}$ remained the same. In the meantime we increased the number of parameters by $3\left(w_{k+1}, \log T_{k+1}\right.$ and $\left.\sigma_{(k+1)}\right)$. Applying Eq. (3) to both $L_{k \max }$ and $L_{(k+1) \max }$ we get after subtraction

$2\left(L_{(k+1) \max }-L_{k \max }\right) \approx \chi_{3}^{2}$.

For $k=1 L_{2 \max }$ is greater than $L_{1 \max }$ by more than 30 , which gives for $\chi_{3}^{2}$ an extremely low probability of $5.88 \times 10^{-13}$. This means that the two log-normal fit is really a better approximation for the duration distribution of GRBs than one log-normal. 


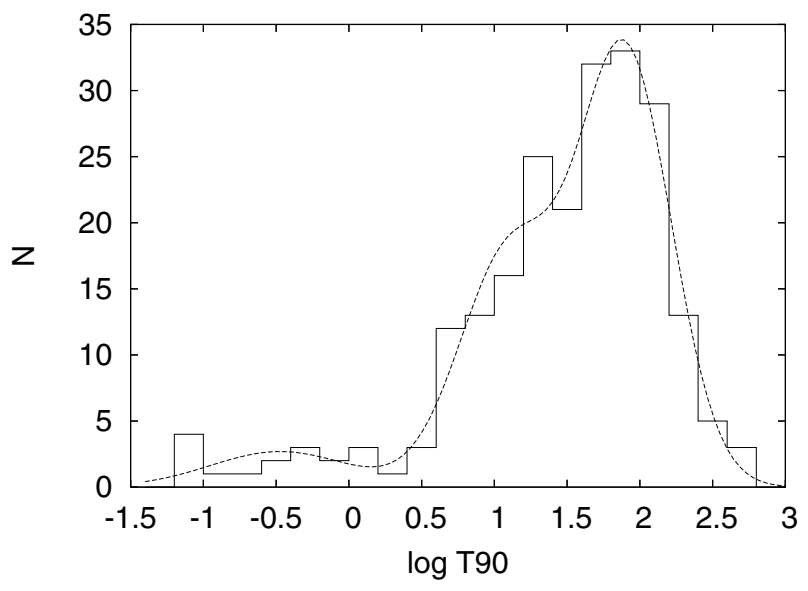

Fig. 3. Fit with three log-normal component for the duration distribution of BAT bursts.

Table 2. The best parameters for the three log-normal fit of the GRB duration distribution.

\begin{tabular}{cccc}
\hline \hline & Duration $\left(\log T_{90}\right)$ & $\sigma\left(\log T_{90}\right)$ & $w$ \\
\hline short & -0.473 & 0.48 & 16.2 \\
long & 1.903 & 0.32 & 129.1 \\
intermediate & 1.107 & 0.35 & 76.7 \\
\hline
\end{tabular}

Table 3. The improvement of the likelihood and the significancies.

\begin{tabular}{cccc}
\hline \hline$i$ & $L_{i \max }$ & $L_{i \max }-L_{(i-1) \max }$ & $p$ \\
\hline 2 & 983.317 & & \\
3 & 989.822 & 6.505 & 0.9954 \\
4 & 990.323 & 0.501 & 0.200 \\
\hline
\end{tabular}

Thirdly, a three-log-normal fit was made combining three $f_{k}$ functions with eight parameters (three means, three standard deviations and two weights). For the best fit parameters see Table 2. The highest value of the logarithm of the likelihood $\left(L_{3 \max }\right)$ is 989.822 . For two log-normal functions the maximum was $L_{2 \max }=983.317$. The maximum thus improved by 6.505 . Twice this is 13.01 which gives us the probability of $0.461 \%$ for the difference between $L_{2 \max }$ and $L_{3 \max }$ is being only by chance. Therefore there is only a small chance the third log-normal is not needed. Thus, the three-log-normal fit (see Fig. 3) is better and there is a 0.0046 probability that it was caused only by statistical fluctuation.

One should also calculate the likelihood for four log-normal functions. The best logarithm of the ML is 990.323. It is bigger by 0.501 than it was with three log-normal functions. This gives us a low significance $(80.1 \%)$, therefore the fourth component is not needed. In Table 3. we summarize the improvement of the likelihood and the probabilities they give us.

\section{1000 Monte-Carlo simulations using the two-component fit}

We can check the 0.0046 probability, which we get for the maximum likelihood calculation, using a Monte-Carlo (MC) simulation and adopting the following procedure. Take the two-log-normal distribution with the best fitted parameters of the observed data, and generate 222 numbers for $T_{90}$ whose distribution follows the two-log-normal distribution. Then find the best likelihood with five free parameters (two means, two
Table 4. Mean hardness ratios and their standard errors.

\begin{tabular}{ccccc}
\hline \hline Type & $\log H 43$ & std. err. $\log H 43$ & $\log H 32$ & std. err. $\log H 32$ \\
\hline short & -0.023 & 0.023 & 0.276 & 0.030 \\
intermediate & -0.185 & 0.014 & 0.112 & 0.018 \\
long & -0.092 & 0.011 & 0.184 & 0.012 \\
\hline
\end{tabular}

dispersions and two weights; but the sum of the last two must be 222). Next we perform a fit with the three-log-normal distribution (eight free parameters, three means, three dispersions and two independent weights). Finally, we take the difference between the two logarithms of the maximum likelihoods that gave one number in our MC simulation.

We have carried out this procedure for 1000 simulations each with 222 simulated $\log T_{90}$ s. There were 8 cases when the $\log$ likelihood difference was more than the one obtained for the BAT data (6.505). Therefore the MC simulations confirm the result obtained by applying Eq. (7) and give a similar $(0.8 \%)$ probability that a third group is merely a statistical fluctuation.

\section{Discussion}

It is possible that the fit using three log-normal functions is accidental, and that there are only two types of GRBs. However, the probability that the third component is only a statistical fluctuation is $0.5-0.8 \%$.

One can compare the burst group weights with previous results. BAT sensitivity is different to BATSE sensitivity (Fishman et al. 1994; Band 2003). BAT is more sensitive at low energies which means it can observe more X-ray flashes and soft bursts and probably fails to detect many hard bursts (typically short ones). Therefore one expects more long and intermediate bursts and fewer short GRBs. In the BAT data set there are only a few short bursts. Our analysis could only find 16 short bursts (7\%). The robustness of the ML method is demonstrated here because a group with only $7 \%$ weight is identified. Previously in the BATSE database intermediate bursts were identified by many research groups. However, in this class different frequencies were found representing 15-25\% of BATSE GRBs (Mukherjee et al. 1998; Hakkila et al. 2000; Balastegui et al. 2001; Rajaniemi \& Mähönen 2002; Horváth et al. 2006).

We calculated the mean $\log H 43$ and $\log H 32$ hardness ratios and the standard errors of the groups as given in Table 4. The table demonstrates, as expected from previous studies, that short bursts are the hardest and the intermediate duration group is the softest among the GRBs detected by Swift. Using a t-test (Kendall \& Stuart 1973) the intermediate bursts differ very significantly (99.9\% in both hardnesses) from the other two and the short and the long bursts are also significantly different in $\log H 43(98 \%)$ and $\log H 32(99 \%)$.

Cline et al. (2005) claims that GRBs with $T_{90}<0.1 \mathrm{~s}$ form a separate group. Out of the 222 Swift GRBs used in our analysis only 4 (25\% of the short population) have a duration of $T_{90}<$ $0.1 \mathrm{~s}$. This very low frequency of these very short GRBs does not allow a detailed statistical analysis. Additionally, the Swift satellite is less sensitive to these types of bursts due to trigger criteria (McLean et al. 2004).

\section{Conclusions}

1. Assuming that the $T_{90}$ distribution of the short and long GRBs is log-normal, the probability that the third group is a chance occurance is about $0.5-0.8 \%$. 
2. Although the statistics indicate that a third component is present, the physical existence of the third group is still debatable. The sky distribution of the third component is anisotropic as proven by Mészáros et al. (2000) and Litvin et al. (2001). Alternatively Hakkila et al. (2000) believe the third statistically proven subgroup is only a deviation caused by complicated instrumental effects, which can reduce the duration of some faint long bursts. This paper does not deal with this particular effect, however the previously studied BATSE sample shows a similar group structure. This agreement suggests that the third component is possibly real, not an instrumental effect (the BATSE detectors and the Swift BAT are different kinds of instruments).

3. The observed frequencies in the three classes are different for BATSE and BAT. Both samples are dominated, however, by the long bursts. The short bursts are less populated in BAT than in BATSE but the intermediate group is more numerous. This is understandable, since BAT is less sensitive in high energy than BATSE was and more sensitive in low energy and short bursts are the hardest group and intermediate ones are the softest. Therefore BAT can observe more intermediate bursts and much fewer short ones than BATSE did.

4. The existence and physical properties of the intermediate group need further discussion to elucidate the reality and properties of this class of GRBs.

Acknowledgements. This research was supported in part through OTKA T048870 grant and Bolyai Scholarship (I.H.). We thank R. J. Nemiroff, the referee, for useful comments.

\section{References}

Bagoly, Z., Mészáros, A., Balázs, L. G., et al. 2006, A\&A, 453, 797

Balastegui, A., Ruiz-Lapuente, P., \& Canal, R. 2001, MNRAS, 328, 283

Balázs, L. G., Bagoly, Z., Horváth, I., Mészáros, A., \& Mészáros, P. 2003, A\&A, 401, 129

Band, D. L. 2003, ApJ, 588, 945
Belli, B. M. 1997, ApJ, 479, L31

Borgonovo, L. 2004, A\&A, 418, 487

Chattopadhyay, T., Misra, R., Chattopadhyay, A. K., \& Naskar, M. 2007, ApJ, 667, 1017

Cline, D. B., Czerny, B., Matthey, C., Janiuk, A., \& Otwinowski, S. 2005, ApJ, 633, L73

Fishman, G. J., Meegan, C. A., Wilson, R. B., et al. 1994, ApJS, 92, 229

Fox, D. B., Frail, D. A., Price, P. A., et al. 2005, Nature, 437, 845

Hakkila, J., Haglin, D. J., Pendleton, G. N., et al. 2000, ApJ, 538, 165

Hakkila, J., Giblin, T. W., Roiger, R. J., et al. 2003, ApJ, 582, 320

Hakkila, J., Giblin, T. W., Roiger, R. J., et al. 2004, Baltic Astron., 13, 211

Horváth, I. 1998, ApJ, 508, 757

Horváth, I. 2002, A\&A, 392, 791

Horváth, I., Balázs, L. G., Bagoly, Z., Ryde, F., \& Mészáros, A. 2006, A\&A, 447, 23

Kendall, M., \& Stuart, A. 1973, The Advanced Theory of Statistics (London \& High Wycombe: Charles Griffin \& Co. Ltd.)

Koshut, T. M., Paciesas, W. S., Kouveliotou, C., et al. 1996, ApJ, 463, 570

Kouveliotou, C., Meegan, C. A., Fishman, G. J., et al. 1993, ApJ, 413, L101

Kouveliotou, C., Koshut, T., Briggs, M. S., et al. 1996, in Amer. Instit. Phys. Conf. Ser. 384, ed. C. Kouveliotou, M. F. Briggs, \& G. J. Fishman, 42

Litvin, V. F., Matveev, S. A., Mamedov, S. V., \& Orlov, V. V. 2001, Astron. Lett., 27, 416

Magliocchetti, M., Ghirlanda, G., \& Celotti, A. 2003, MNRAS, 343, 255

Mazets, E. P., Golenetskii, S. V., Ilinskii, V. N., et al. 1981, Ap\&SS, 80, 3

McBreen, B., Hurley, K. J., Long, R., \& Metcalfe, L. 1994, MNRAS, 271, 662

McLean, K. M., Fenimore, E. E., Palmer, D., et al. 2004, in Gamma-Ray Bursts: 30 Years of Discovery, ed. E. Fenimore, \& M. Galassi, Amer. Instit. Phys. Conf. Ser., 727, 667

Meegan, C. A., Pendleton, G. N., Briggs, M. S., et al. 1996, ApJS, 106, 65

Mészáros, A., Bagoly, Z., Horváth, I., Balázs, L. G., \& Vavrek, R. 2000, ApJ, 539,98

Mukherjee, S., Feigelson, E. D., Babu, G. J., et al. 1998, ApJ, 508, 314

Norris, J. P., Cline, T. L., Desai, U. D., \& Teegarden, B. J. 1984, Nature, 308, 434

Norris, J. P., Scargle, J. D., \& Bonnell, J. T. 2001, in Gamma-ray Bursts in the Afterglow Era, ed. E. Costa, F. Frontera, \& J. Hjorth, 40

O’Shaughnessy, R., Belczynski, K., \& Kalogera, V. 2008, ApJ, 675, 566

Pendleton, G. N., Paciesas, W. S., Briggs, M. S., et al. 1997, ApJ, 489, 175

Rajaniemi, H. J., \& Mähönen, P. 2002, ApJ, 566, 202

Sakamoto, T., Barthelmy, S. D., Barbier, L., et al. 2008, ApJS, 175, 179

Vanderspek, R., Dullighan, A., Butler, N., et al. 2004, in Gamma-Ray Bursts: 30 Years of Discovery, ed. E. Fenimore, \& M. Galassi, Amer. Instit. Phys. Conf. Ser., 727, 57 